\title{
Aplicativo de Quiz sobre Química Inorgânica acessível a pessoas com deficiência visual: QuiSalino
}

\author{
Priscila Thais Vaz ${ }^{1}$, Aline Alves Ramos², Siralberto Souza Leitão de Almeida ${ }^{3}$ \\ ${ }^{1}$ Centro de Matemática, Computação e Cognição - Universidade Federal do ABC (ABC) \\ ${ }^{2}$ Centro de Ciência Naturais e Humanas - Universidade Federal do ABC (ABC) \\ ${ }^{3} \mathrm{MBA}$ - Faculdade de Informática e Administração Paulista (FIAP) \\ ${ }^{1,2}$ Av. dos Estados, 5001 - Bangu - Santo André \\ ${ }^{3}$ Av. Lins de Vasconcelos, 1222 - Aclimação - São Paulo/SP \\ \{priscila.vaz@ufabc.edu.br, linearamos@gmail.com, contato@dev- \\ sirox.com.br\}
}

Resumo. Este artigo tem como objetivo propor um objeto de aprendizagem do tipo jogo com finalidade de atingir pessoas com deficiência visual (PcDV). Apesar do grande potencial das tecnologias assistivas utilizadas por pessoas com deficiência, ainda encontram-se desafios principalmente relacionado a conteúdos inacessíveis. A matéria escolhida foi a de Química por ter conteúdos de difícil acesso as PcDV, como imagens, fórmulas, gráficos e estruturas. A preocupação com o desenvolvimento de diretrizes capazes de auxiliar na elaboração de objetos de aprendizagem acessíveis vem de encontro a necessidade de inclusão de PcDV a Educação, seja a distância ou presencial.

\section{Cenário de Uso}

Com base em revisão sistemática, que investigou acessibilidade na educação, um dos maiores problemas encontrados é a elaboração de conteúdos acessíveis, estas dificuldades estão presentes em diversas áreas do conhecimento, porém, Química foi selecionada, pois muitas são as dificuldades encontradas entre elas a quantidade de informações a serem memorizadas e também a necessidade frequente de utilizar recursos visuais para interpretar gráficos, desenhos, modelos e estruturas (SANTOS, 2004).

De acordo com Retondo e Silva (2008), há uma grande falta de conexão entre práticas de ensino de Química e ensino-aprendizagem de pessoas com deficiência visual (PcDV), pois pouca se tem desenvolvido em relação a livros, apostilas, softwares e aplicativos inclusivos.

Santos e Paulino (2006) indicam que muito se tem a realizar no âmbito da inclusão educacional. Dentre os sentidos, a visão é o mais requerido no ensino-aprendizado de Química, surge então o desafio de adaptar conteúdos a deficientes visuais. 
V Congresso Brasileiro de Informática na Educação (CBIE 2016)

Anais dos Workshops do V Congresso Brasileiro de Informática na Educação (CBIE 2016)

Segundo Santarosa et al. (2009), é fundamental quesitos que envolvam acessibilidade no desenvolvimento de recursos pedagógicos. No caso de objetos de aprendizagem, possibilita aos professores, desenvolverem estratégias de mediação, possibilitando a inclusão social e digital. (SANTAROSA \& BASSO, 2009 e CAMARGO-FILHO \& BICA, 2009).

Entretanto, somente o uso de tecnologias assistivas não são suficientes para tornar objetos de aprendizagem acessíveis, é necessário que os seus conteúdos sejam criados e disponibilizados segundo os padrões de acessibilidade.

O tema Sais, abordado em Química Inorgânica, foi selecionado por ser pré-requisito para outros tópicos abordados na disciplina, além de fazer parte do cotidiano do aluno, possibilitando a contextualização.

O objetivo geral foi propor um objeto de aprendizagem acessível capaz de auxiliar estudantes, com deficiência visual, de ensino médio e graduação a compreender o contexto e os conceitos químicos de nomenclatura e formulação de sais inorgânicos, afim de auxiliar alunos e professores e consequentemente promover a inclusão.

\section{Desenvolvimento}

O desenvolvimento do projeto foi realizado com a linguagem de marcação HTML5, Linguagem de programação JavaScript/Jquery, CSS para estilos, utilizando as ferramentas IDE (Integrated Development Environment) Intel XDK para desenvolvimento do mesmo JustMind para criação da simulação do protótipo. A linguagem HTML5, apresenta alterações que facilitam a acessibilidade, como possibilidade de aplicar semântica em áreas estruturais de uma página, suporte para funcionalidades multimídia por controles capazes de serem acionados por usuários de teclado, possibilitando aos desenvolvedores criarem controles de mídia personalizados.

Como principal elemento, foi utilizado também o Plugin chamado phonegap-pluginmobile-accessibility (GITHUB, 2016), que permite um aplicativo enviar algo para ser anunciada pelo leitor de tela, também um comando para parar o leitor de tela, informações sobre o status de vários recursos de acessibilidade aos sistemas operacionais móveis, incluindo, por exemplo, se um leitor de tela está em execução, inverter cores caso esteja ativado, e a escalabilidade preferida para o texto. 
V Congresso Brasileiro de Informática na Educação (CBIE 2016)

Anais dos Workshops do V Congresso Brasileiro de Informática na Educação (CBIE 2016)

Se tratando de Banco de Dados, foi aplicado o uso de dados nas nuvens com o serviço chamado Firebase (FIREBASE, 2016) com parcerias como Google, uma nova maneira de gerenciar projetos, com armazenamento de arquivos, notificações, Analystics gratuito em dispositivos móveis, fornece consumo em tempo real, promete evolução de suas ferramentas e facilidade aos desenvolvedores backend.

\section{Apresentação do Software}

A proposta do aplicativo acessível em formato de jogo do tipo Quiz, utilizando o assunto abordado em Química Inorgânica: Sais, figura 01.

No jogo, o aluno tem acesso a introdução do conteúdo, com sua contextualização e depois um quiz de perguntas a respeito de nomenclatura e formulação dos sais, o jogo conta com níveis de dificuldades que se encontra em desenvolvimento.

Para tornar o aplicativo acessível, além da preocupação com a forma de abordagem do conteúdo, imagens, fórmulas, botões e alternativas são todas descritivas para que o leitor de tela conseguisse passar a informação necessária para a pessoa com deficiência visual.

O projeto, como mencionado na seção 2, foi desenvolvido em HTML5, possibilitando o desenvolvimento de aplicativos para dispositivos móveis e Web, assim o projeto poderá ser disponibilizado tanto para celulares como para tablets, segundo figura 02.

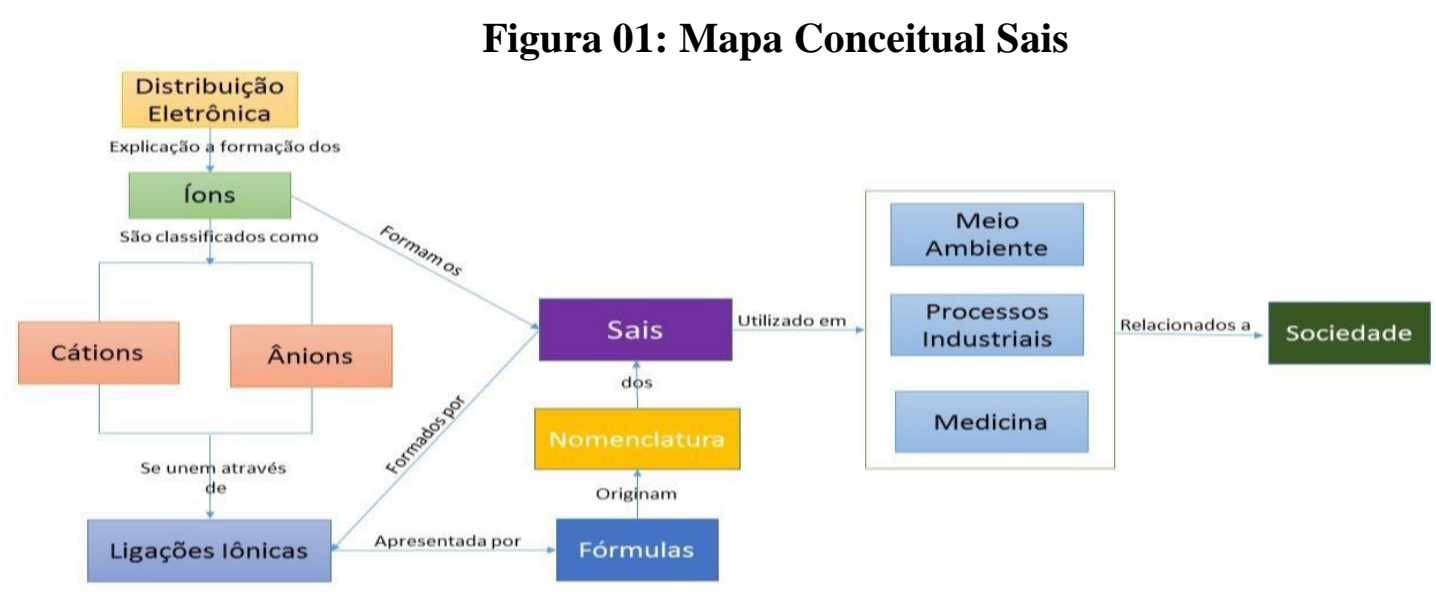

Fonte: Autoria 
V Congresso Brasileiro de Informática na Educação (CBIE 2016)

Anais dos Workshops do V Congresso Brasileiro de Informática na Educação (CBIE 2016)

\section{Figura 02: Mapa Conceitual Aplicativo}

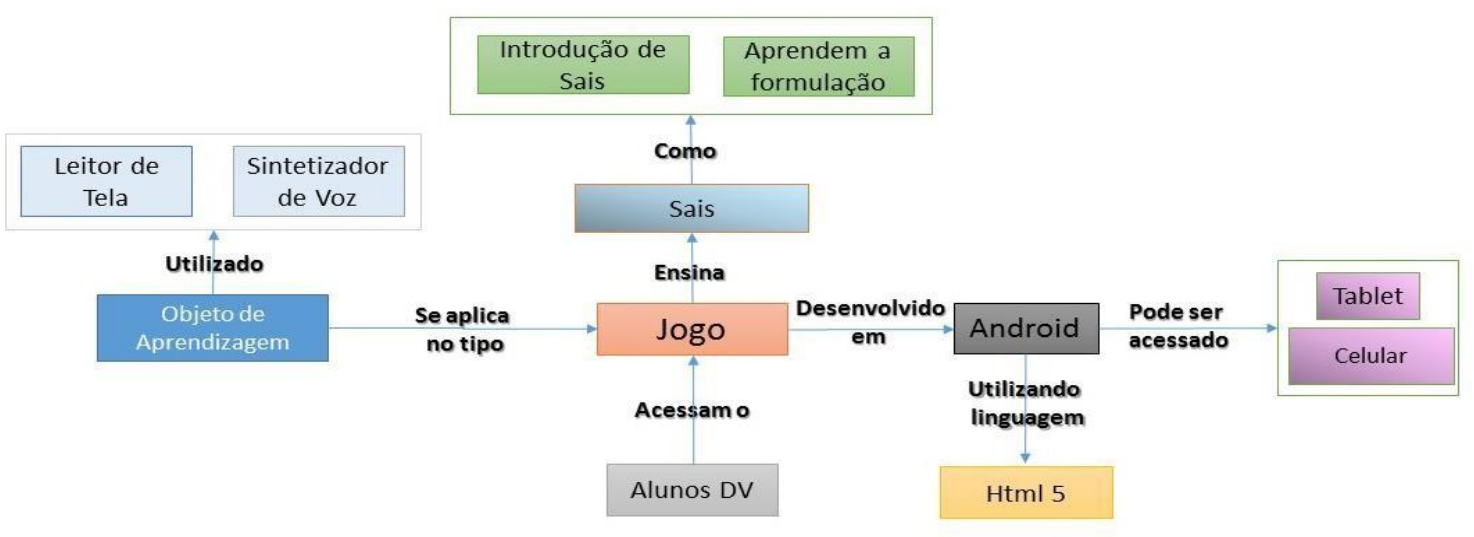

Fonte: Autoria

Para melhor entendimento e visualização do aplicativo basta acessar o link para o vídeo demonstrativo do aplicativo: https://youtu.be/OFAzlXEDDlQ, também é possível testa - ló em seu celular ou tablet Android acessando o link:

https://drive.google.com/file/d/0B6sYAiUa54jdT0VwOUx5VTISZHc/view?usp=sharin g

Como descrição das telas, algumas das principais telas são apresentadas. Na figura 03.

Figura 03: Introdução a Sais

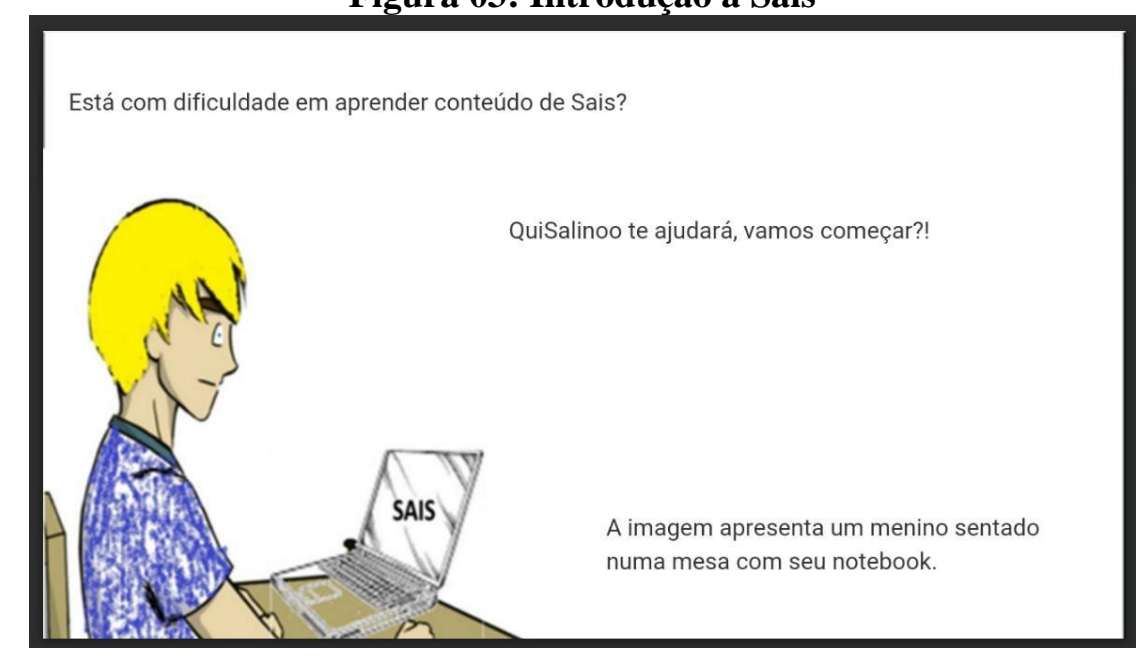

Na figura 03 e 04 são apresentadas as telas de contextualização de sais, para melhor acessibilidade com o leitor de tela, as fórmulas foram descritas no código, com objetivo de não poluir a tela para pessoas sem deficiência acessarem. 
V Congresso Brasileiro de Informática na Educação (CBIE 2016)

Anais dos Workshops do V Congresso Brasileiro de Informática na Educação (CBIE 2016)

Figura 03: Introdução a Sais

\section{Definição}

Sais são substâncias formadas pela neutralização de um ácido com uma base.

Exemplo:

União do ácido clorídrico $(\mathrm{HCl})$ com hidróxido de sódio $(\mathrm{NaOH})$ forma cloreto de sódio $(\mathrm{NaCl})$ e água $\left(\mathrm{H}^{2} \mathrm{O}\right)$.

Lembrando que ácido libera $\mathrm{H}+$ (aga mais) mais água e base libera $\mathrm{OH}-(\mathrm{O}$ aga menos).

Sendo assim, SAIS são qualquer substância inorgânica que em água libera pelo menos um cátion diferente de $\mathrm{H}+$ e pelo menos um ânion diferente de $\mathrm{OH}$ -

Figura 04: Introdução a Sais

Alguns exemplos de Sais

Sal de cozinha é o cloreto de sódio $(\mathrm{NaCl})$.

Além do "sal de cozinha", os sais estão presentes na composição de vários produtos que utilizamos no dia a dia.

O fluoreto de sódio ( $\mathrm{NaF}$ ), por exemplo, é empregado em pastas de dente como fonte de flúor, elemento químico de grande importância no combate às cáries.

Se você ou alguém que conhece já sofreu um acidente em que precisou imobilizar alguma parte do corpo com gesso, saiba que o maior parte dele é constituída por um sal chamado sulfato de cálcio (CaSO4).

Na figura 05 é apresentada a tela como introdução para prática, ou seja, o início do jogo de quiz.

Figura 05: Vamos praticar?!

Vamos Praticar?

Clique Duas Vezes Na Tela Para Começar O Sorteio.

Na figura 06 é apresentada a continuação do início do jogo onde ao clicar duas vezes na tela, uma fórmula será sorteada. 
V Congresso Brasileiro de Informática na Educação (CBIE 2016)

Anais dos Workshops do V Congresso Brasileiro de Informática na Educação (CBIE 2016)

Figura 06: Sorteio

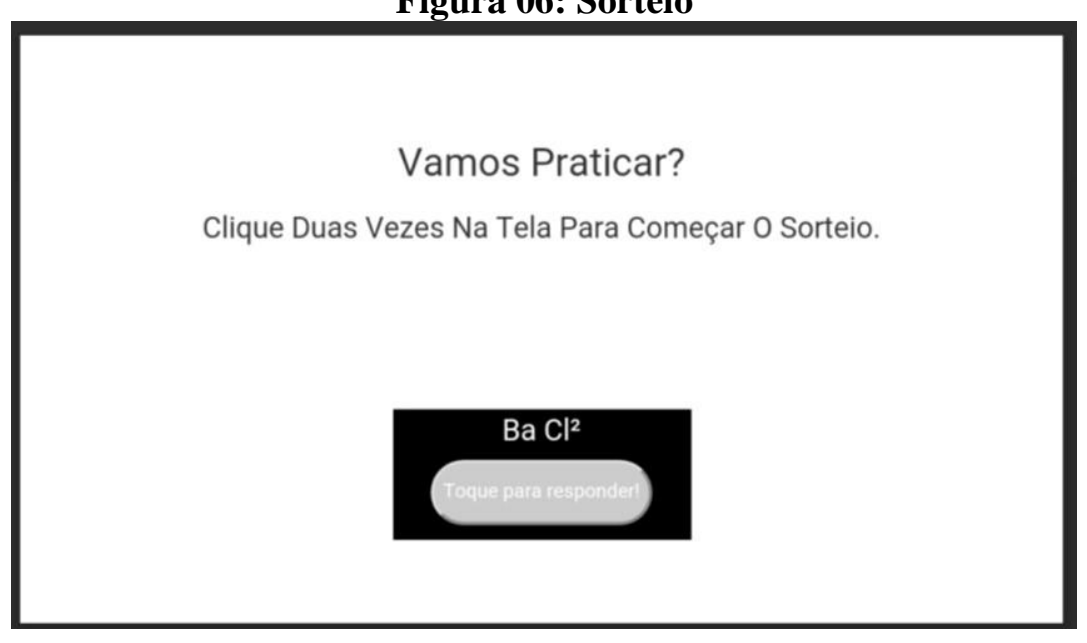

Na figura 07 é apresentado a tela com as alternativas, tendo somente uma alternativa correta, ao clicar em cada alternativa, o leitor de tela passa a informação para o deficiente visual e o mesmo pode escolher a resposta que julgar correta.

Figura 07: Tela Fórmulas

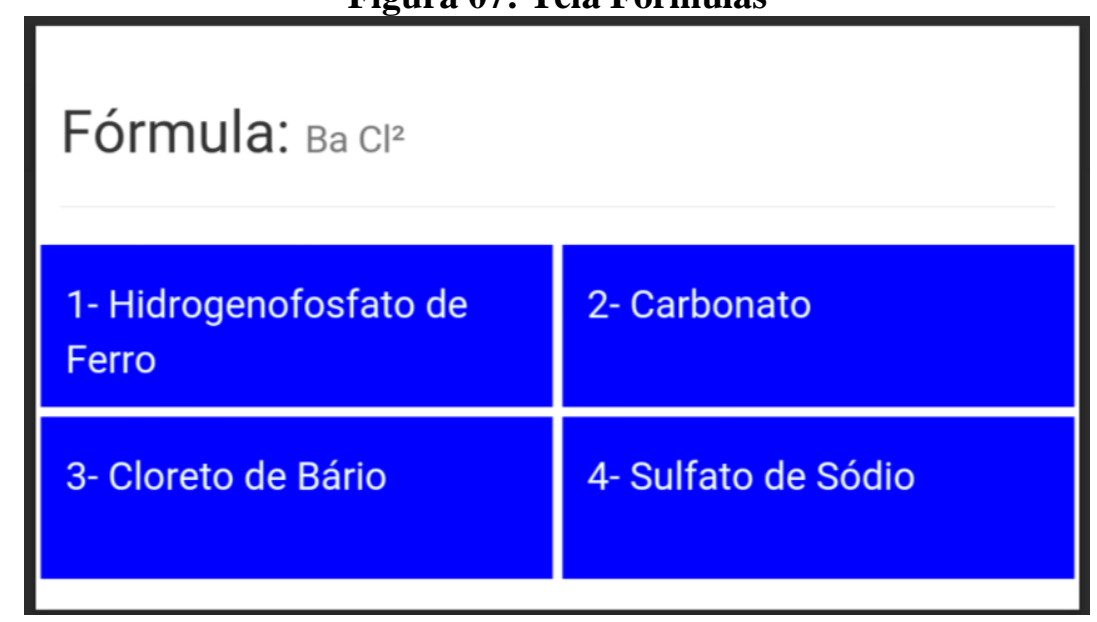

Na figura 08 e 09 é apresentado a tela do feedback de acordo com a resposta do aluno, caso correto, o jogador recebe feedback positivo podendo retornar para um novo sorteio, caso a resposta seja incorreta, feedback de negativo será apresentado e uma nova fórmula será sorteada. 
V Congresso Brasileiro de Informática na Educação (CBIE 2016)

Anais dos Workshops do V Congresso Brasileiro de Informática na Educação (CBIE 2016)

Figura 08: Feedback positivo

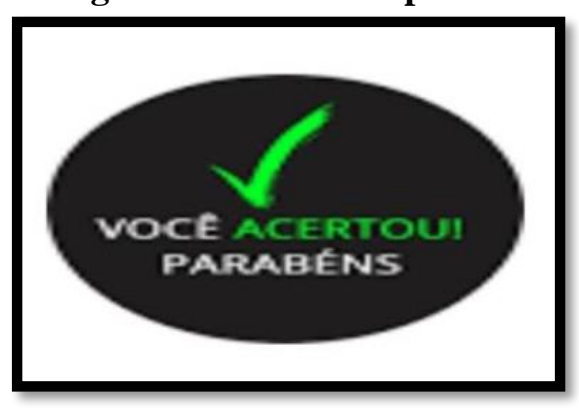

Figura 09: Feedback negativo

\section{Considerações finais}

Em busca de soluções que abrangessem questões de problemas enfrentados na educação por alunos com deficiência visual, foi sugerido a utilização de metodologia de desenvolvimento de OA acessível, tendo como exemplo a criação de um jogo em Química.

Com estudos levantados foi possível verificar que mesmo com a utilização de Tecnologias Assistivas, ainda existe falta de acessibilidade em conteúdo para os deficientes visuais, impedindo-os muitas vezes de continuar seus estudos.

Com o trabalho foi possível analisar e intervir em diretrizes para serem utilizadas no desenvolvimento do objeto de aprendizagem acessíveis.

Como perspectivas futura tem-se a possibilidade de validação com pessoas deficientes visuais e novas ideias a serem incrementas no aplicativo já desenvolvido e disponibilização para utilização no Google Play. 
V Congresso Brasileiro de Informática na Educação (CBIE 2016)

Anais dos Workshops do V Congresso Brasileiro de Informática na Educação (CBIE 2016)

\section{Referência}

FIREBASE. Disponível em: https://firebase.googleblog.com/2016/05/firebase-expands- tobecome-unified-app-platform.html. Acesso em 25 de maio de 2016.

GITHUB. Disponível em: https://github.com/phonegap/phonegap-mobile-accessibility. Acesso em 15 de maio de 2016.

RETONDO, C. G. \& SILVA, G. M. Ressignificação a Formação de Professores de Química para a Educação Especial e inclusiva: Uma História de Parceria. Química Nova, no 30, p. 27-33, 2008.

SANTAROSA, Lucila M. C; CONFORTO, D.; BASSO L. O. AVA inclusivo: validação da acessibilidade na perspectiva de interagentes com limitações visuais e auditivas. In XVIII Simpósio Brasileiro de Informática na Educação, 2009. SBIE.

SANTAROSA, L.M.C; BASSO, L.O. 2009. Multimediaworkshop: collective production in learning management systems with the aim of PSN digital inclusion. IN: WORLD CONFERENCE ON COMPUTER IN EDUCATION. World Conference on Computer in Education - WCCE09, 2009, Bento Gonçalves. Anais... Bento Gonçalves.

SANTOS, W. L. P. Educação em Química: compromisso com a cidadania. Editora Ijuí, 3 ed, 2004.

SANTOS, M. P; PAULINO, M. M. Inclusão em educação: Culturas, políticas e práticas. São Paulo: Cortez, 2006. 